TRANSACTIONS OF THE

AMERICAN MATHEMATICAL SOCIETY

Volume 364, Number 10, October 2012, Pages 5491-5507

S 0002-9947(2012)05539-5

Article electronically published on May 7, 2012

\title{
A LIMIT $q=-1$ FOR THE BIG $q$-JACOBI POLYNOMIALS
}

\author{
LUC VINET AND ALEXEI ZHEDANOV
}

\begin{abstract}
We study a new family of "classical" orthogonal polynomials, here called big -1 Jacobi polynomials, which satisfy (apart from a 3-term recurrence relation) an eigenvalue problem with differential operators of Dunkl type. These polynomials can be obtained from the big $q$-Jacobi polynomials in the limit $q \rightarrow-1$. An explicit expression of these polynomials in terms of Gauss' hypergeometric functions is found. The big -1 Jacobi polynomials are orthogonal on the union of two symmetric intervals of the real axis. We show that the big -1 Jacobi polynomials can be obtained from the (terminating) Bannai-Ito polynomials when the orthogonality support is extended to an infinite number of points. We further indicate that these polynomials provide a nontrivial realization of the Askey-Wilson algebra for $q \rightarrow-1$.
\end{abstract}

\section{INTRODUCTION}

We constructed in [19] a system of "classical" orthogonal polynomials $P_{n}(x)$ containing two real parameters $\alpha, \beta$ and corresponding to the limit $q \rightarrow-1$ of the little $q$-Jacobi polynomials. By "classical" we mean that these polynomials satisfy (apart from a 3-term recurrence relation) a nontrivial eigenvalue equation of the form

$$
L P_{n}(x)=\lambda_{n} P_{n}(x) .
$$

The novelty lies in the fact that $L$ is a differential-difference operator of special type. Namely, $L$ is a linear operator which is of first order in the derivative operator $\partial_{x}$ and also contains the reflection operator $R$ which acts as $R f(x)=f(-x)$. Roughly speaking, one can say that $L$ belongs to the class of Dunkl operators 6 which contain both the operators $\partial_{x}$ and $R$. Nevertheless, the operator $L$ differs from the standard Dunkl operators in a fundamental way. Indeed, $L$ preserves the linear space of polynomials of any given maximal degree. This basic property allows us to construct a complete system of polynomials $P_{n}(x), n=0,1,2, \ldots$ as eigenfunctions of the operator $L$.

Guided by the $q \rightarrow-1$ limit of the little $q$-Jacobi polynomials, we derived in [19] an explicit expression of the polynomials $P_{n}(x)$ in terms of Gauss' hypergeometric functions. We also found explicitly the recurrence coefficients and showed that the polynomials $P_{n}(x)$ are orthogonal on the interval $[-1,1]$ with a weight function related to the weight function of the generalized Jacobi polynomials [5. We also proved that they admit the Dunkl classical property [3] and further demonstrated

Received by the editors November 29, 2010 and, in revised form, December 23, 2010, January 3, 2011, January 5, 2011, and January 8, 2011.

2010 Mathematics Subject Classification. Primary 33C45, 33C47, 42C05.

Key words and phrases. Classical orthogonal polynomials, Jacobi polynomials, big $q$-Jacobi polynomials. 
that the operator $L$ together with the multiplication operator $x$ forms a special case of the Askey-Wilson algebra $\mathrm{AW}(3)$ [20] corresponding to the parameter $q=-1$.

In this paper we similarly construct a new family of "classical" orthogonal polynomials which are obtained as a nontrivial limit of the big $q$-Jacobi polynomials when $q \rightarrow-1$. We will call them "big -1 Jacobi polynomials".

In contrast to the little -1 Jacobi polynomials, the big -1 Jacobi polynomials contain 3 real parameters $\alpha, \beta, c$. This leads to more complicated formulas for the recurrence coefficients as well as for the explicit expression in terms of the Gauss hypergeometric function. Moreover, in contrast to the little -1 Jacobi polynomials the big -1 Jacobi polynomials are orthogonal on the union of the two intervals $[-1,-c]$ and $[c, 1]$ (it is assumed that $0<c<1$ ). When $c=0$ these intervals connect into one interval $[-1,1]$. This corresponds to the degeneration of the big -1 Jacobi polynomials into the little -1 Jacobi polynomials

The fundamental "classical" property (1.1) holds for the big -1 Jacobi polynomials as well. The operator $L$ is again a first order differential operator of Dunkl type which preserves the space of polynomials. This means that both little and big -1 Jacobi polynomials provide two "missing" families of classical orthogonal polynomials which should be included into the Askey table as special cases.

The first nontrivial example of explicit polynomials corresponding to the limit $q \rightarrow-1$ in the Askey scheme was proposed by Bannai and Ito [2], 18]. The terminating Bannai-Ito polynomials are orthogonal on a finite set of $N+1$ points. The nonterminating Bannai-Ito polynomials (corresponding to the direct limit $N \rightarrow \infty$ ) are not positive definite. We show nevertheless that the big -1 Jacobi polynomials are obtained in some special limit $N \rightarrow \infty$ from the Bannai-Ito polynomials in a way that preserves positive definiteness.

We also demonstrate that the big -1 Jacobi polynomials provide a convenient realization of the $\mathrm{AW}(3)$ algebra for $q=-1$.

\section{Big $q$-JACOBI POLYNOMIALS IN The Limit $q=-1$}

The big $q$-Jacobi polynomials $P_{n}(x ; a, b, c)$ were introduced by Andrews and Askey [1] as an infinite-dimensional version of the $q$-Hahn polynomials. Implicitly, the big $q$-Jacobi polynomials are also contained in the Bannai-Ito scheme of dual systems of orthogonal polynomials as an infinite dimension analogue of the $q$-Racah polynomials [2]. These polynomials depend on 3 parameters $a, b, c$ and are defined by the following 3 -term recurrence relation (for brevity, we will sometimes omit the dependence on the parameters $a, b, c)$ :

$$
P_{n+1}(x)+b_{n} P_{n}(x)+u_{n} P_{n-1}(x)=x P_{n}(x),
$$

where

$$
u_{n}=A_{n-1} C_{n}, \quad b_{n}=1-A_{n}-C_{n}
$$

with

$$
\begin{aligned}
A_{n} & =\frac{\left(1-a q^{n+1}\right)\left(1-a b q^{n+1}\right)\left(1-c q^{n+1}\right)}{\left(1-a b q^{2 n+1}\right)\left(1-a b q^{2 n+2}\right)} \\
C_{n} & =-a c q^{n+1} \frac{\left(1-q^{n}\right)\left(1-a b c^{-1} q^{n}\right)\left(1-b q^{n}\right)}{\left(1-a b q^{2 n+1}\right)\left(1-a b q^{2 n}\right)} .
\end{aligned}
$$


In terms of basic hypergeometric functions [10, [1] they are given by

$$
P_{n}(x ; a, b, c)=\kappa_{n 3} \varphi_{2}\left(\begin{array}{c}
q^{-n}, a b q^{n+1}, x \\
a q, c q
\end{array} \mid q ; q\right),
$$

where the coefficient $\kappa_{n}$ ensures that $P_{n}(x)$ is monic: $P_{n}(x)=x^{n}+O\left(x^{n-1}\right)$. We shall not need the explicit expression of $\kappa_{n}$ in the following.

The big $q$-Jacobi polynomials satisfy the eigenvalue equation [10], [1]

$$
L P_{n}(x)=\lambda_{n} P_{n}(x), \quad \lambda_{n}=\left(q^{-n}-1\right)\left(1-a b q^{n+1}\right),
$$

where the operator $L$ is

$$
L f(x)=B(x)(f(x q)-f(x))+D(x)\left(f\left(x q^{-1}\right)-f(x)\right)
$$

with

$$
B(x)=\frac{a q(x-1)(b x-c)}{x^{2}}, \quad D(x)=\frac{(x-a q)(x-c q)}{x^{2}} .
$$

The orthogonality relation is

$$
\int_{c q}^{a q} w(x) P_{n}(x) P_{m}(x) d_{q} x=h_{n} \delta_{n m}, \quad h_{n}=u_{1} u_{2} \ldots u_{n},
$$

with the $q$-integral defined as [10, 11]

$$
\int_{c q}^{a q} f(x) d_{q} x=a q(1-q) \sum_{s=0}^{\infty} f\left(a q^{s+1}\right) q^{s}-c q(1-q) \sum_{s=0}^{\infty} f\left(c q^{s+1}\right) q^{s}
$$

and the weight function

$$
w(x)=g \frac{\left(a^{-1} x ; q\right)_{\infty}\left(c^{-1} x ; q\right)_{\infty}}{(x ; q)_{\infty}\left(b c^{-1} x ; q\right)_{\infty}},
$$

where

$$
(a ; q)_{s}=(1-a)(1-a q) \ldots\left(1-a q^{s-1}\right)
$$

is the shifted $q$-factorial [11] and $(a ; q)_{\infty}=\lim _{s \rightarrow \infty}(a ; q)_{s}$. (In (2.8), $g$ is a normalization factor which is not essential for our considerations.)

Consider the operator $(q+1)^{-1} L$, where the operator $L$ is defined by (2.5). Put

$$
q=-\exp (\epsilon), a=-\exp (\epsilon \alpha), b=-\exp (\epsilon \beta)
$$

and take the limit $\epsilon \rightarrow 0$ which corresponds to the limit $q \rightarrow-1$. It is not difficult to verify that the limit does exist and that we have

$$
L_{0}=\lim _{q \rightarrow-1}(q+1)^{-1} L=g_{0}(x)(R-I)+g_{1}(x) \partial_{x} R,
$$

where

$$
g_{0}(x)=\frac{(\alpha+\beta+1) x^{2}+(c \alpha-\beta) x+c}{x^{2}}, \quad g_{1}(x)=\frac{2(x-1)(x+c)}{x} .
$$

The operator $I$ is the identity operator and $R$ is the reflection operator $R f(x)=$ $f(-x)$.

Equivalently, the operator $L_{0}$ can be presented through its action on $f(x)$ :

$$
L_{0} f(x)=g_{0}(x)(f(-x)-f(x))-g_{1}(x) f^{\prime}(-x) .
$$

On monomials $x^{n}$ the operator $L_{0}$ acts as follows.

For $n$ even,

$$
L_{0} x^{n}=4 n(x-1)(x+c) x^{n-2} .
$$


For $n$ odd,

$$
L_{0} x^{n}=-2(\alpha+\beta+n+1) x^{n}+2(\beta-c \alpha+n-c) x^{n-1}+2(n-1) c x^{n-2} .
$$

In any case, the operator $L_{0}$ is lower triangular, with 3 diagonals, in the basis $x^{n}$ :

$$
L x^{n}=\xi_{n} x^{n}+\eta_{n} x^{n-1}+\zeta_{n} x^{n-2}
$$

with the coefficients $\xi_{n}, \eta_{n}, \zeta_{n}$ straightforwardly obtained from (2.13), (2.14). It is easily seen that the operator $L_{0}$ preserves the linear space of polynomials of any fixed dimension. Hence for every $n=0,1,2, \ldots$ there are monic polynomial eigenfunctions $P_{n}^{(-1)}(x)=x^{n}+O\left(x^{n-1}\right)$ of the operator $L_{0}$.

This eigenvalue equation is obtained as the $q \rightarrow-1$ limit of the eigenvalue equation (2.4):

$$
L_{0} P_{n}^{(-1)}(x)=\lambda_{n} P_{n}^{(-1)}(x)
$$

where

$$
\lambda_{n}=\left\{\begin{array}{c}
2 n, \quad n \text { even, } \\
-2(\alpha+\beta+n+1), \quad n \text { odd. }
\end{array}\right.
$$

Consider the limit $q \rightarrow-1$ for the recurrence coefficients. Assuming (2.9), we have

$$
A_{n}^{(-1)}=\lim _{\epsilon \rightarrow 0} A_{n}=\left\{\begin{array}{lll}
\frac{(c+1)(\alpha+n+1)}{\alpha+\beta+2 n+2}, & n & \text { even, } \\
\frac{(1-c)(\alpha+\beta+n+1)}{\alpha+\beta+2 n+2}, & n & \text { odd }
\end{array}\right.
$$

and

$$
C_{n}^{(-1)}=\lim _{\epsilon \rightarrow 0} C_{n}=\left\{\begin{array}{ccc}
\frac{(1-c) n}{\alpha+\beta+2 n}, & n & \text { even, } \\
\frac{(1+c)(\beta+n)}{\alpha+\beta+2 n}, & n & \text { odd. }
\end{array}\right.
$$

Hence for the recurrence coefficients we have

$$
u_{n}^{(-1)}=\lim _{\epsilon \rightarrow 0} A_{n-1} C_{n}=\left\{\begin{array}{lll}
\frac{(1-c)^{2} n(\alpha+\beta+n)}{(\alpha+\beta+2 n)^{2}}, & n & \text { even }, \\
\frac{(1+c)^{2}(\alpha+n)(\beta+n)}{(\alpha+\beta+2 n)^{2}}, & n & \text { odd }
\end{array}\right.
$$

and

$$
b_{n}^{(-1)}=\lim _{\epsilon \rightarrow 0} 1-A_{n}-C_{n}=\left\{\begin{array}{ccc}
-c+\frac{(c-1) n}{\alpha+\beta+2 n}+\frac{(1+c)(\beta+n+1)}{\alpha+\beta+2 n+2}, & n & \text { even } \\
c+\frac{(1-c)(n+1)}{\alpha+\beta+2 n+2}-\frac{(c+1)(\beta+n)}{\alpha+\beta+2 n}, & n & \text { odd }
\end{array}\right.
$$

The polynomials $P_{n}^{(-1)}(x)$ satisfy the 3 -term recurrence relation

$$
P_{n+1}^{(-1)}(x)+b_{n}^{(-1)} P_{n}^{(-1)}(x)+u_{n}^{(-1)} P_{n-1}^{(-1)}(x)=x P_{n}^{(-1)}(x) .
$$

For any real $c \neq 1$ and real $\alpha, \beta$ satisfying the restriction $\alpha>-1, \beta>-1$, the recurrence coefficients $b_{n}^{(-1)}$ are real and the recurrence coefficients $u_{n}$ are positive. This means that the polynomials $P_{n}^{(-1)}(x)$ are positive definite orthogonal polynomials.

Let us consider expression (2.3) in detail,

$$
P_{n}(x)=\kappa_{n} \sum_{s=0}^{n} \frac{\left(q^{-n} ; q\right)_{s}\left(a b q^{n+1} ; q\right)_{s}(x ; q)_{s}}{(q ; q)_{s}(a q ; q)_{s}(c q ; q)_{s}} q^{s}
$$


In the limit $q \rightarrow-1$ it is easy to obtain that

$$
\frac{(x ; q)_{s}}{(c q ; q)_{s}}=\left\{\begin{array}{ccl}
\left(\frac{1-x^{2}}{1-c^{2}}\right)^{s / 2}, & s & \text { even }, \\
\frac{1-x}{1+c}\left(\frac{1-x^{2}}{1-c^{2}}\right)^{(s-1) / 2}, & s & \text { odd }
\end{array}\right.
$$

Hence, in the limit $q \rightarrow-1$ the sum (2.23) is divided into two parts. The first part is an even polynomial with respect to $x$, i.e. $p\left(x^{2}\right)$, where $p(x)$ is a polynomial. The second part will have the form $(1-x) q\left(x^{2}\right)$ with another polynomial $q(x)$. Simple calculations lead to the following formulas.

If $n$ is even,

$$
\begin{aligned}
& P_{n}^{(-1)}(x)=\kappa_{n}\left[{ } _ { 2 } F _ { 1 } \left(-\frac{n}{2}, \frac{n+\alpha+\beta+2}{2}\left|\frac{1-x^{2}}{\frac{\alpha+1}{2}}\right|\right.\right.
\end{aligned}
$$

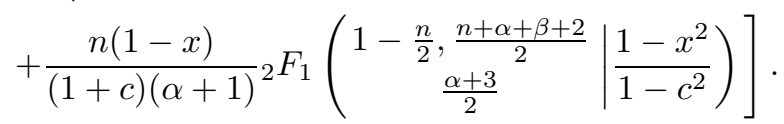

If $n$ is odd,

$$
\begin{aligned}
P_{n}^{(-1)}(x)=\kappa_{n}\left[{ }_{2}\right. & F_{1}\left(\begin{array}{c}
\left.-\frac{n-1}{2}, \frac{n+\alpha+\beta+1}{2} \mid \frac{1-x^{2}}{1-c^{2}}\right) \\
\frac{\alpha+1}{2}
\end{array}\right. \\
& \left.\quad-\frac{(\alpha+\beta+n+1)(1-x)}{(1+c)(\alpha+1)}{ }_{2} F_{1}\left(-\frac{n-1}{2}, \frac{n+\alpha+\beta+3}{2} \mid \frac{1-x^{2}}{1-c^{2}}\right)\right] .
\end{aligned}
$$

The normalization coefficient is given by

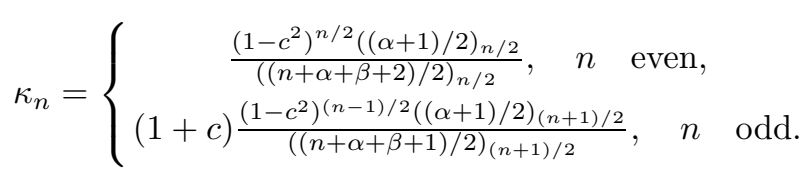

The remaining problem is to find the orthogonality relation and the corresponding weight function $w(x)$ for the big -1 Jacobi polynomials. Of course, this could be done directly from the known orthogonality relation for the big $q$-Jacobi polynomials by taking the limit $q \rightarrow-1$. However it is more instructive to derive the weight function using the method of polynomial mappings [8], [15]. This method will allow us to find nontrivial relations between the big -1 Jacobi polynomials and the ordinary Jacobi polynomials. This will explain the origin of the rather "strange" expressions (2.24) and (2.25).

\section{Polynomial systems and the Christoffel transform}

In this section we consider a scheme allowing us to obtain a new family of orthogonal polynomials starting from two sets of orthogonal polynomials related by the Christoffel transform. This scheme is a simple generalization of the well-known Chihara method for constructing symmetric orthogonal polynomials from a pair of orthogonal polynomials and their kernel partner [4]. It is also very close to the scheme proposed by Marcellán and Petronilho in [15]. 
Let $P_{n}(x), n=0,1,2, \ldots$ be a set of monic orthogonal polynomials satisfying the recurrence relation

$$
P_{n+1}(x)+b_{n} P_{n}(x)+u_{n} P_{n-1}(x)=x P_{n}(x) .
$$

Consider a partner family of orthogonal polynomials $Q_{n}(x)$ related to $P_{n}(x)$ by the Christoffel transform 16

$$
Q_{n}(x)=\frac{P_{n+1}(x)-A_{n} P_{n}(x)}{x-\nu^{2}}
$$

where $\nu$ is a real parameter and $A_{n}=P_{n+1}\left(\nu^{2}\right) / P_{n}\left(\nu^{2}\right)$.

If the polynomials $P_{n}(x)$ are monic orthogonal with respect to the linear functional $\sigma$,

$$
\left\langle\sigma, P_{n}(x) P_{m}(x)\right\rangle=0, \quad n \neq m,
$$

then the polynomials $Q_{n}(x)$ are monic orthogonal with respect to the functional $\tilde{\sigma}=\left(x-\nu^{2}\right) \sigma$, i.e. 16 .

$$
\left\langle\sigma,\left(x-\nu^{2}\right) Q_{n}(x) Q_{m}(x)\right\rangle=0, \quad n \neq m .
$$

The polynomials $P_{n}(x)$ are expressed in terms of the polynomials $Q_{n}(x)$ via the Geronimus transform 21

$$
P_{n}(x)=Q_{n}(x)-B_{n} Q_{n-1}(x),
$$

where the coefficients $B_{n}$ are related to $A_{n}$ and the recurrence coefficients by the formulas

$$
u_{n}=B_{n} A_{n-1}, \quad b_{n}=-A_{n}-B_{n}+\nu^{2} .
$$

Now, starting from a pair of polynomials $P_{n}(x), Q_{n}(x)$ we can construct another family of orthogonal polynomial $R_{n}(x)$ by proceeding as follows.

For even numbers $n$, let the polynomials $R_{n}(x)$ be defined according to

$$
R_{2 n}(x)=P_{n}\left(x^{2}\right)
$$

and for odd numbers $n$, let

$$
R_{2 n+1}(x)=(x-\nu) Q_{n}\left(x^{2}\right) .
$$

It is obvious that for all $n=0,1,2, \ldots$ the polynomials $R_{n}(x)$ are monic polynomials in $x$ of degree $n$.

What is more important is that the polynomials $R_{n}(x)$ are orthogonal, since they satisfy the 3 -term recurrence relation

$$
R_{n+1}(x)+(-1)^{n} \nu R_{n}(x)+v_{n} R_{n-1}(x)=x R_{n}(x),
$$

where

$$
v_{2 n}=-B_{n}, v_{2 n+1}=-A_{n} .
$$

This construction can also be carried out in reverse.

Assuming that the polynomials $R_{n}(x)$ satisfy the recurrence relation (3.7) with some real parameter $\nu$ and positive coefficients $v_{n}$, it can easily be shown by induction that

$$
R_{2 n}(x)=P_{n}\left(x^{2}\right), \quad R_{2 n+1}(x)=(x-\nu) Q_{n}\left(x^{2}\right),
$$

where $P_{n}(x), Q_{n}(x)$ are monic polynomials of degree $n$. 
The polynomials $R_{n}(x)$ are orthogonal with respect to a positive definite linear functional $\rho$ :

$$
\left\langle\rho, R_{n}(x) R_{m}(x)\right\rangle=0, \quad n \neq m .
$$

Let

$$
r_{n}=\left\langle\rho, x^{n}\right\rangle
$$

be the corresponding moments. We use the standard normalization condition $r_{0}=$ 1. It can then be proven, again by induction, that

$$
r_{2 n+1}=\nu r_{2 n}, \quad n=0,1,2, \ldots
$$

and that the even moment $r_{2 n}$ is an even monic polynomial of degree $2 n$ in the argument $\nu$, i.e.

$$
r_{2 n}=\nu^{2 n}+n v_{1} \nu^{2 n-2}+\frac{n(n-1)}{2} v_{1}\left(v_{1}+v_{2}\right) \nu^{2 n-4}+O\left(\nu^{2 n-6}\right) .
$$

It is directly verified that the polynomials $P_{n}(x)$ and $Q_{n}(x)$ are orthogonal as they satisfy the recurrence relations

$$
P_{n+1}(x)+\left(v_{2 n}+v_{2 n+1}+\nu^{2}\right) P_{n}(x)+v_{2 n} v_{2 n-1} P_{n-1}(x)=x P_{n}(x)
$$

and

$$
Q_{n+1}(x)+\left(v_{2 n+2}+v_{2 n+1}+\nu^{2}\right) Q_{n}(x)+v_{2 n} v_{2 n+1} Q_{n-1}(x)=x Q_{n}(x) .
$$

Moreover, the polynomials $Q_{n}(x)$ are Christoffel transforms of the polynomials $P_{n}(x)$ :

$$
Q_{n}(x)=\frac{P_{n+1}(x)+v_{2 n+1} P_{n}(x)}{x-\nu^{2}},
$$

while the polynomials $P_{n}(x)$ are Geronimus transforms of $Q_{n}(x)$ :

$$
P_{n}(x)=Q_{n}(x)+v_{2 n} Q_{n-1}(x) .
$$

Assume that the polynomials $P_{n}(x)$ have moments $c_{n}$. Then one has a simple relation between the moments

$$
r_{2 n}=c_{n}, r_{2 n+1}=\nu c_{n}, \quad n=0,1,2, \ldots .
$$

The moments $\tilde{c}_{n}$ corresponding to the polynomials $Q_{n}(x)$ are given by

$$
\tilde{c}_{n}=\frac{c_{n+1}-\nu^{2} c_{n}}{c_{1}-\nu^{2}} .
$$

Expression (3.12) follows easily from the definition of the Christoffel transform [21].

Note that in the special case $\nu=0$ we recover the well-known scheme relating symmetric and nonsymmetric polynomials that has been described in detail by Chihara [4. In this case the polynomials $R_{n}(x)$ are symmetric, $R_{n}(-x)=(-1)^{n} R_{n}(x)$, and their odd moments are zero, $r_{2 n+1}=0$. All the above formulas remain valid if one puts $\nu=0$. We have thus provided a generalization of the Chihara scheme with an additional parameter $\nu$. Note that the resulting polynomials $R_{n}(x)$ are no longer symmetric; however they satisfy the simple recurrence relation (3.7) and have properties very close to those of symmetric orthogonal polynomials.

In [15] a more general problem was studied with the orthogonal polynomials $R_{n}(x)$ defined as $R_{2 n}(x)=P_{n}(\phi(x))$, where $\phi(x)$ is a polynomial of second degree and $P_{n}(x)$ is a given system of orthogonal polynomials. Our approach corresponds to the special case $\phi(x)=x^{2}$. Note that the general case of a polynomial mapping has the form $R_{N n}(x)=P_{n}\left(\pi_{N}(x)\right)$, where $\pi_{N}(x)$ is a polynomial of degree $N$. 
Again it is assumed that both $P_{n}(x)$ and $R_{n}(x)$ are nondegenerate orthogonal polynomials. The theory of such mappings was considered in 8 .

Now consider the following concrete example connected with Jacobi polynomials. This example will allow us to establish the weight function of the big-1-Jacobi polynomials.

Let

$$
P_{n}^{(\xi, \eta)}(x)=G_{n 2} F_{1}\left(\begin{array}{c}
-n, n+\xi+\eta+1 \\
\xi+1
\end{array} ; x\right)
$$

be Jacobi polynomials with the orthogonality relation

$$
\int_{0}^{1} x^{\xi}(1-x)^{\eta} P_{n}^{(\xi, \eta)}(x) P_{m}^{(\xi, \eta)}(x) d x=h_{n} m \delta_{n m}
$$

on the interval $[0,1]$.

The normalization coefficient

$$
G_{n}=(-1)^{n} \frac{(\xi+1)_{n}}{(n+\xi+\eta+1)_{n}}
$$

ensures that $P_{n}(x)$ is monic: $P_{n}^{(\xi, \eta)}(x)=x^{n}+O\left(x^{n-1}\right)$.

First perform an affine transformation of the argument and consider the new monic orthogonal polynomials

$$
P_{n}(x)=\left(c^{2}-1\right)^{n} P_{n}^{(\xi, \eta)}\left(\frac{1-x}{1-c^{2}}\right),
$$

where $c$ is a real parameter with the restriction $0<c<1$.

In terms of hypergeometric functions,

$$
P_{n}(x)=\left(1-c^{2}\right)^{n} \frac{(\xi+1)_{n}}{(n+\xi+\eta+1)_{n}}{ }_{2} F_{1}\left(\begin{array}{c}
-n, n+\xi+\eta+1 \\
\xi+1
\end{array} ; \frac{1-x}{1-c^{2}}\right) .
$$

Clearly, these polynomials are orthogonal on the interval $\left[c^{2}, 1\right]$,

$$
\int_{c^{2}}^{1}(1-x)^{\xi}\left(x-c^{2}\right)^{\eta} P_{n}(x) P_{m}(x) d x=0, \quad n \neq m .
$$

Also introduce the companion polynomials $Q_{n}(x)$ through the Christoffel transform

$$
Q_{n}(x)=\frac{P_{n+1}(x)-A_{n} P_{n}(x)}{x-1}, \quad A_{n}=\frac{P_{n+1}(1)}{P_{n}(1)} .
$$

It is easily seen that the polynomials $Q_{n}(x)$ are again expressible in terms of Jacobi polynomials with $\xi \rightarrow \xi+1$ :

$$
Q_{n}(x)=\left(c^{2}-1\right)^{n} P_{n}^{(\xi+1, \eta)}\left(\frac{1-x}{1-c^{2}}\right),
$$

or, in terms of hypergeometric functions,

$$
Q_{n}(x)=\left(1-c^{2}\right)^{n} \frac{(\xi+2)_{n}}{(n+\xi+\eta+2)_{n}}{ }_{2} F_{1}\left(\begin{array}{c}
-n, n+\xi+\eta+2 \\
\xi+2
\end{array} ; \frac{1-x}{1-c^{2}}\right) .
$$

The polynomials $P_{n}(x)$ and $Q_{n}(x)$ are connected by the relations (3.2) and (3.3) with $\nu=1$. The coefficients $A_{n}$ and $B_{n}$ can be found from the following observation. Putting $x=1$, we find from (3.13) and (3.15),

$$
P_{n}(1)=\left(1-c^{2}\right)^{n} \frac{(\xi+1)_{n}}{(n+\xi+\eta+1)_{n}}, \quad Q_{n}(1)=\left(1-c^{2}\right)^{n} \frac{(\xi+2)_{n}}{(n+\xi+\eta+2)_{n}} .
$$


From these formulas we immediately get

$$
A_{n}=\frac{P_{n+1}(1)}{P_{n}(1)}=\left(1-c^{2}\right) \frac{(\xi+n+1)(\xi+\eta+n+1)}{(2 n+\xi+\eta+1)(2 n+\xi+\eta+2)}
$$

and

$$
B_{n}=\frac{Q_{n}(1)-P_{n}(1)}{Q_{n-1}(1)}=\left(1-c^{2}\right) \frac{n(\eta+n)}{(2 n+\xi+\eta)(2 n+\xi+\eta+1)} .
$$

Note that $A_{n}>0, B_{n}>0$ for $n=1,2, \ldots$ due to the restriction $0<c<1$.

Now consider the new monic orthogonal polynomials $R_{n}(x)$ defined by the relations

$$
R_{2 n}(x)=P_{n}\left(x^{2}\right), \quad R_{2 n+1}(x)=(x-1) Q_{n}\left(x^{2}\right) .
$$

According to the general theory of polynomial mappings [8, 15, it is not difficult to show that the polynomials $R_{n}(x)$ are orthogonal on a domain formed by the union of two intervals $[-1,-c],[c, 1]$ of the real axis:

$$
\int_{-1}^{-c} R_{n}(x) R_{m}(x) W(x) d x+\int_{c}^{1} R_{n}(x) R_{m}(x) W(x) d x=0, \quad n \neq m,
$$

where the (nonnormalized) weight function is:

$$
W(x)=\theta(x)(1+x)\left(1-x^{2}\right)^{\xi}\left(x^{2}-c^{2}\right)^{\eta}
$$

and $\theta(x)=x /|x|$ is the sign function. Note that the weight function $W(x)$ is not positive on the interval $[-1,-c]$.

In terms of Gauss' hypergeometric functions we have the expressions

$$
R_{2 n}(x)=P_{n}\left(x^{2}\right)=\left(1-c^{2}\right)^{n} \frac{(\xi+1)_{n}}{(n+\xi+\eta+1)_{n}}{ }_{2} F_{1}\left(\begin{array}{c}
-n, n+\xi+\eta+1 \\
\xi+1
\end{array} ; \frac{1-x^{2}}{1-c^{2}}\right)
$$

and

$$
\begin{aligned}
R_{2 n+1}(x) & =(x-1) Q_{n}\left(x^{2}\right) \\
& =\left(1-c^{2}\right)^{n} \frac{(\xi+2)_{n}}{(n+\xi+\eta+2)_{n}}(x-1)_{2} F_{1}\left(\begin{array}{c}
-n, n+\xi+\eta+2 \\
\xi+2
\end{array} ; \frac{1-x^{2}}{1-c^{2}}\right) .
\end{aligned}
$$

The polynomials $R_{n}(x)$ satisfy the 3 -term recurrence relation

$$
R_{n+1}(x)+(-1)^{n} R_{n}(x)+v_{n} R_{n-1}(x)=x R_{n}(x),
$$

where

$$
\begin{gathered}
v_{2 n}=-B_{n}=\left(c^{2}-1\right) \frac{n(\eta+n)}{(2 n+\xi+\eta)(2 n+\xi+\eta+1)}, \\
v_{2 n+1}=-A_{n}=\left(c^{2}-1\right) \frac{(\xi+n+1)(\xi+\eta+n+1)}{(2 n+\xi+\eta+1)(2 n+\xi+\eta+2)} .
\end{gathered}
$$

Note that all the coefficients $v_{n}$ are negative, $v_{n}<0$, which corresponds to the nonpositivity of the weight function $W(x)$. 


\section{The Weight Function And the orthogonality OF THE BIG -1 JACOBI POLYNOMIALS}

In order to determine the weight function and the orthogonality region for the big -1 Jacobi polynomials, we notice that formulas (2.24) and (2.25) can be presented in the following equivalent form:

$$
P_{n}^{(-1)}(x)=R_{n}(x)-G_{n} R_{n-1}(x),
$$

where $R_{n}(x)$ are the polynomials defined by (3.21), (3.22) that satisfy the recurrence relation (3.23). In these formulas we should put $\xi=(\alpha-1) / 2, \eta=(\beta+1) / 2$. The coefficients $G_{n}$ have the expression

$$
G_{n}=\left\{\begin{array}{ccc}
\frac{(1-c) n}{2 n+\alpha+\beta}, & n & \text { even, } \\
-\frac{(1+c)(n+\alpha)}{2 n+\alpha+\beta}, & n & \text { odd } .
\end{array}\right.
$$

It is well known that if two families of orthogonal polynomials are related by a formula such as (4.1), then necessarily the polynomials $P_{n}^{(-1)}(x)$ are obtained from the polynomials $R_{n}(x)$ by the Geronimus transform [21]. This is equivalent to the statement that the weight function $w^{(-1)}(x)$ of the polynomials $P_{n}^{(-1)}(x)$ is obtained from the weight function $W(x)$ of the polynomials $R_{n}(x)$ as follows:

$$
w^{(-1)}(x)=\frac{W(x)}{x-\mu}+M \delta(x-\mu),
$$

with two additional parameters $\mu$ and $M$. Formula (4.3) means that apart from the division of the weight function $W(x)$ by the linear factor $x-\mu$ there is an additional concentrated mass $M$ that is inserted at the point $x=\mu$.

The parameter $\mu$ can be found from the recurrence relation for the coefficients $G_{n}[21$,

$$
G_{n+1}+(-1)^{n}+\frac{v_{n}}{G_{n}}=\mu,
$$

with the recurrence coefficients $v_{n}$ given by (3.24).

Substituting (4.2) into (4.4) we obtain $\mu=-c$.

Thus the orthogonality relation for polynomials $P_{n}^{(-1)}(x)$ takes the form

$$
\int_{\Gamma} P_{n}^{(-1)}(x) P_{m}^{(-1)}(x) W(x)(x+c)^{-1} d x+M P_{n}^{(-1)}(-c) P_{m}^{(-1)}(-c)=0, \quad n \neq m,
$$

where the contour $\Gamma$ is the union of the two intervals $[-1,-c]$ and $[c, 1]$ of the real axis.

In order to find the value $M$ of the concentrated mass it is sufficient to consider a special case of the orthogonality relation (4.5) for $n=1, m=0$,

$$
\int_{\Gamma} P_{1}^{(-1)}(x) W(x)(x+c)^{-1} d x+M P_{1}(-c)=0 .
$$

Now, $P_{1}^{(-1)}(x)$ is given by

$$
P_{1}^{(-1)}(x)=x+\zeta
$$

where $\zeta=\frac{c(\alpha+1)-\beta-1}{2+\alpha+\beta}$. Substituting this expression into (4.6) and calculating the integral (through an elementary reduction to the Euler beta-integral) we find that $M=0$. 
Thus, the orthogonality relation for polynomials $P_{n}^{(-1)}(x)$ reads

$$
\int_{\Gamma} P_{n}^{(-1)}(x) P_{m}^{(-1)}(x) w^{(-1)}(x) d x=0, \quad n \neq m,
$$

where the weight function $w^{(-1)}(x)$ can be presented in the form

$$
w^{(-1)}(x)=\theta(x)(x+1)(x+c)^{-1}\left(1-x^{2}\right)^{(\alpha-1) / 2}\left(x^{2}-c^{2}\right)^{(\beta+1) / 2}
$$

or, equivalently,

$$
w^{(-1)}(x)=\theta(x)(x+1)(x-c)\left(1-x^{2}\right)^{(\alpha-1) / 2}\left(x^{2}-c^{2}\right)^{(\beta-1) / 2} .
$$

Note that under the restrictions $\alpha>-1, \beta>-1$, the weight function is positive on the two intervals of $\Gamma$ and all the moments

$$
m_{n}=\int_{\Gamma} w^{(-1)}(x) x^{n} d x
$$

are finite for $n=0,1,2, \ldots$

When $c \rightarrow 0$, the big $q$-Jacobi polynomials reduce to the little $q$-Jacobi polynomials [10]. In the limit case $q \rightarrow-1$ we see that when $c \rightarrow 0$, the set of two intervals coalesces to the single interval $[-1,1]$ and the weight function becomes

$$
\left.w(x)\right|_{c=0}=(1+x)|x|^{\beta}\left(1-x^{2}\right)^{(\alpha-1) / 2},
$$

which corresponds to the weight function of the little -1 Jacobi polynomials [19.

So far, we considered the case $0<c<0$. The case $c>1$ can be treated in an analogous way. This leads to the following orthogonality relation:

$$
\int_{-c}^{-1} P_{n}^{(-1)}(x) P_{m}^{(-1)}(x) w^{(-1)}(x) d x+\int_{1}^{c} P_{n}^{(-1)}(x) P_{m}^{(-1)}(x) w^{(-1)}(x) d x=0, \quad n \neq m,
$$

where the weight function is almost the same as in (4.9) with obvious modifications:

$$
w^{(-1)}(x)=\theta(x)(x+1)(c-x)\left(x^{2}-1\right)^{(\alpha-1) / 2}\left(c^{2}-x^{2}\right)^{(\beta-1) / 2}
$$

and where again we have the restrictions $\alpha>-1, \beta>-1$.

The case $c=1$ is degenerate: the recurrence coefficients $u_{n}$ for even $n$ become zero, $u_{2 n}=0$, which means that orthogonal polynomials $P_{n}^{(1)}(x)$ are no longer positive definite. The two intervals of orthogonality shrink into two points $x= \pm 1$.

\section{The BIG -1 JaCobi POlynomials as limits OF THE BANNAI-ITO POLYNOMIALS}

Bannai and Ito proved a theorem which characterizes all dual polynomial schemes [2]. This theorem is a generalization of the Leonard theorem [14, where only the finite-dimensional case was considered.

As part of this characterization Bannai and Ito found polynomials (see below) that are not contained in the list of known explicit orthogonal polynomials of the Askey scheme. They are related to the limit $q \rightarrow-1$ of the $q$-Racah polynomials. These can be either terminating $(N<\infty)$ or nonterminating $(N=\infty)$.

The terminating Bannai-Ito polynomials $W_{n}(x)$ are specified as follows: they are orthogonal on a finite number of points $x_{i}, i=0,1,2, \ldots, N$ given by

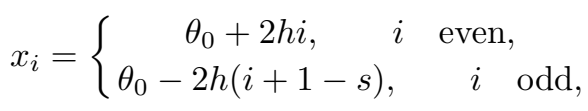

where $\theta_{0}, h, s$ are arbitrary parameters. 
The polynomials $W_{n}(x)$ satisfy the recurrence relation [2], 18]

$$
A_{n} W_{n+1}(x)+\left(\theta_{0}-A_{n}-C_{n}\right) W_{n}(x)+C_{n} W_{n-1}(x)=x W_{n}(x),
$$

where

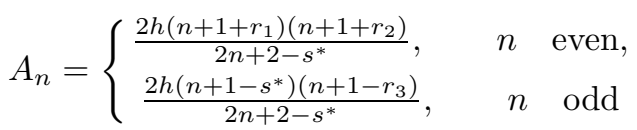

and

$$
C_{n}=\left\{\begin{array}{ccc}
-\frac{2 h n\left(n-s^{*}+r_{3}\right)}{2 n-s^{*}}, & n \text { even, } \\
-\frac{2 h\left(n-r_{1}-s^{*}\right)\left(n-r_{2}-s^{*}\right)}{2 n-s^{*}}, & n \text { odd },
\end{array}\right.
$$

where $s^{*}, r_{1}, r_{2}$ are parameters such that

$$
s+s^{*}=r_{3}-r_{1}-r_{2}
$$

and $r_{2}=-N-1$ if $N$ is even and $r_{3}=N+1$ if $N$ is odd.

The polynomials $W_{n}(x)$ are not monic. Instead, they satisfy the conditions [2] $W_{n}\left(\theta_{0}\right)=1, n=0,1,2, \ldots, N$. As usual, it is assumed that $W_{-1}(x)=0$. We can introduce the monic Bannai-Ito polynomials $\hat{W}_{n}(x)=x^{n}+O\left(x^{n-1}\right)$ by the formula

$$
\hat{W}_{n}(x)=A_{0} A_{1} \ldots A_{n-1} W_{n}(x) .
$$

The polynomials $\hat{W}_{n}(x)$ are then seen to satisfy the recurrence relation

$$
\hat{W}_{n+1}(x)+\left(\theta_{0}-A_{n}-C_{n}\right) \hat{W}_{n}(x)+A_{n-1} C_{n} \hat{W}_{n-1}(x)=x \hat{W}_{n}(x) .
$$

Note that the parameter $\theta_{0}$ is not essential: it only specifies the initial point $x_{0}$. We can thus put $\theta_{0}=1$ without loss of generality. The recurrence relation (5.6) then determines all polynomials $\hat{W}_{n}(x)$ uniquely.

The nonterminating Bannai-Ito polynomials $(N=\infty)$ are given by the same formulas (5.1) -(5.5) with the condition below (5.5) removed; thus $h, r_{1}, r_{2}, r_{3}, s$ and $s^{*}$ are free subject only to (5.5). As we shall show below, the nonterminating BannaiIto polynomials are only quasi-definite. We shall first focus on the terminating polynomials $W_{n}(x)$ and examine their limit when $N \rightarrow \infty$ and $h \rightarrow 0$ to establish the connection with the big -1 Jacobi polynomials.

Let us now consider this limit $N \rightarrow \infty$. Given the relations that $r_{2}$ and $r_{3}$ have with $N$, it is clear that under such a limit, we must necessarily have: $r_{3} \rightarrow \infty$ and $r_{2} \rightarrow-\infty$. We assume that the parameters $r_{1}, s^{*}$ are finite. It is now convenient to put

$$
r_{1}=\alpha, \quad s^{*}=-\alpha-\beta
$$

with some fixed parameters $\alpha, \beta$.

From (5.5) it then follows that $s \rightarrow \infty$. The parameter $h$, moreover, is free: it allows for a scaling of the argument $x$. We can thus choose $h \rightarrow 0$ in such a way that

$$
2 h r_{2} \rightarrow c+1, \quad 2 h r_{3} \rightarrow c-1,
$$

where $c$ is a parameter such that $0<c<1$.

Then, in the limit $N \rightarrow \infty$, the recurrence coefficients $A_{n}$ and $C_{n}$ coincide with the coefficients $A_{n}, C_{n}$ given by the formulas (2.18) and (2.19), and we have already observed that these coefficients $A_{n}$ and $C_{n}$ determine uniquely the big -1 Jacobi polynomials. 
Consider the behavior of the argument $x_{i}$ in the limit $N \rightarrow \infty$. As $h \rightarrow 0$, let $i \rightarrow \infty$ in a way such that $2 h i \rightarrow-y$, where $y$ is a continuous variable which varies from 0 to $c$. Hence from (5.1), in the limit $N \rightarrow \infty$, the points $x_{i}$, for even $i$, densely cover the interval $[c, 1]$. Similarly, from (5.5), (5.7),

$$
\lim _{N \rightarrow \infty} 2 h s=\lim _{N \rightarrow \infty}\left\{2 h r_{3}-2 h r_{2}\right\}=-2
$$

and hence from (5.1), in the limit $N \rightarrow \infty$, the points $x_{i}$, for odd $i$, densely cover the interval $[-1,-c]$. This corresponds to the fact that the big -1 Jacobi polynomials are orthogonal on the union of the two intervals $[-1,-c]$ and $[c, 1]$.

We thus have shown that the big -1 Jacobi polynomials can be obtained from the terminating Bannai-Ito polynomials $W_{n}(x)$ through a limit process where $N \rightarrow$ $\infty, h \rightarrow 0$. This complements the fact demonstrated in this paper that the big -1 Jacobi polynomials arise as a $q \rightarrow-1$ limit from the big $q$-Jacobi polynomials.

When we remind ourselves that the big $q$-Jacobi polynomials result from an $N \rightarrow \infty$ limit of the $q$-Racah polynomials [10], we see that we have the following commutative diagram for limit relations between these polynomials:

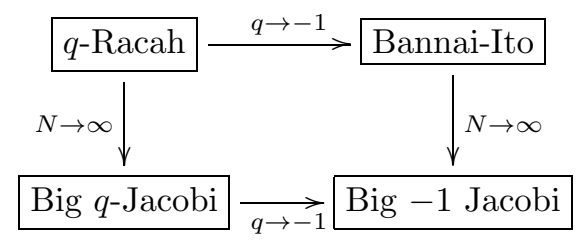

Note finally that that there is an essential difference in the limiting behavior of the $q$-Racah and the Bannai-Ito polynomials when $N \rightarrow \infty$. Indeed, from the $q$-Racah polynomials one obtains the big $q$-Jacobi polynomials which have a purely discrete orthogonality measure, and thus the duality property is preserved. This fact is indicated in [2]. Namely Bannai and Ito have included the possibility of $N=\infty$ for the case of $q$-Racah polynomials, in which limit the duality property is preserved. However the limiting process is not trivial and, in fact, only very recently have details of this limit been studied by Koornwinder [12.

In contrast, for the Bannai-Ito polynomials a nondegenerate positive-definite limiting process $N \rightarrow \infty$ is possible only if $h \rightarrow 0$. We briefly show that positive definite orthogonal Bannai-Ito polynomials do not exist in the limit $N \rightarrow \infty$ if $h$ is finite, thereby demonstrating that the nonterminating Bannai-Ito polynomials are not orthogonal. Indeed, it is well known that the positive definiteness property for orthogonal polynomials means the existence of a positive nondecreasing measure on the real axis with respect to which the polynomials are orthogonal [4. In turn, the positive definiteness is equivalent to the positivity of the product $A_{n-1} C_{n}$ of the recurrence coefficient in relation (5.6) 2. Assume that the constant $h$ is nonzero. Clearly, we can always assume that $h>0$. Assume first that all the parameters $r_{1}, r_{2}, r_{3}, s^{*}$ are finite. Then it is visible from expressions (5.3), (5.4) that for sufficiently large values of $n$ the coefficient $A_{n}$ is positive while the coefficient $C_{n}$ is negative. Hence for sufficiently large $n$ we have $A_{n-1} C_{n}<0$, which means that the positive definite property is violated. The only possibility to get the condition $A_{n-1} C_{n}>0$ is to assume that either $A_{n}$ or $C_{n}$ are truncated for some finite $N$ and hence the positivity condition will hold only for a finite number of points $x_{0}, x_{1}, \ldots, x_{N}$. Further simple analysis shows that the truncation condition is only 
possible for the coefficient $A_{n}$. This gives two possible choices of truncation condition $A_{N}=0$ : either $r_{2}=-N-1$ or $r_{3}=N+1$ depending on the parity of $N$ (of course, one can equally put $r_{1}=-N-1$ in the first case because the numerator of $A_{n}$ for even $n$ is symmetric with respect to $\left.r_{1}, r_{2}\right)$. In this case both $A_{n}$ and $C_{n}$ are negative for $0<n<N+1$ and the positivity property $A_{n-1} C_{n}<0$ holds.

If one assumes that some of the parameters $r_{1}, r_{2}, r_{3}, s^{*}$ are tending to infinity, then it is clear from (5.3) and (5.4) that inevitably $s^{*} \rightarrow \infty$ and simultaneously some of the parameters $r_{1}, r_{2}$ or $r_{3}$ tend to infinity as well. A simple analysis shows that in this case we also have violation of positivity: $A_{n-1} C_{n}<0$ for sufficiently large $N$.

The nonterminating polynomials with $N=\infty$ that are included in the solution of the Bannai-Ito dual polynomial problem are hence only formal orthogonal polynomials in that only $A_{n-1} C_{n} \neq 0$ is satisfied without positivity.

The well-known example of formal orthogonal polynomials is the Bessel polynomials [4 which are quasi-definite. An orthogonality relation for these polynomials can be provided by an integral on the unit circle [4. Other examples of quasidefinite orthogonal polynomials can be obtained from the ordinary Jacobi polynomials if their parameters do not satisfy the standard conditions. In this case the orthogonality relation can be rather complicated (see [13] for details).

Only if it is accompanied by the limit $h \rightarrow 0$ can the limit $N \rightarrow \infty$ preserve positive definiteness of the orthogonal polynomials. This leads to the big -1 Jacobi polynomials. But of course, the duality property is missing in the limit $N \rightarrow \infty$ because the grid $x_{s}$ disappears. Instead we have bispectrality: the big -1 Jacobi polynomials are eigenfunctions of the Dunkl-differential operator (2.12). As far as we know the possibility of such a limiting process (i.e. $N \rightarrow \infty, h \rightarrow 0$ ) preserving the positivity property has not yet been mentioned in the literature.

\section{Anticommutator algebra describing Big - 1 Jacobi polynomials}

The Askey-Wilson polynomials are described by the AW(3)-algebra [20, [17]. Among the different equivalent forms of this algebra, we choose the following one:

$$
X Y-q Y X=\mu_{3} Z+\omega_{3}, \quad Y Z-q Z Y=\mu_{1} X+\omega_{1}, \quad Z X-q X Z=\mu_{2} Y+\omega_{2},
$$

which possesses an obvious symmetry with respect to all 3 operators (see, e.g., 9]).

Here $q$ is a fixed parameter corresponding to the "base" parameter in the $q$ hypergeometric functions defining the Askey-Wilson polynomials [10. The pairs of operators $(X, Y),(Y, Z)$ and $(Z, X)$ play the role of "Leonard pairs" (see [17, [9]).

The Casimir operator

$$
Q=\left(q^{2}-1\right) X Y Z+\mu_{1} X^{2}+\mu_{2} q^{2} Y^{2}+\mu_{3} Z^{2}+(q+1)\left(\omega_{1} X+\omega_{2} q Y+\omega_{3} Z\right)
$$

commutes with all operators $X, Y, Z$.

The constants $\omega_{i}, i=1,2,2$ (together with the value of the Casimir operator $Q$ ) define representations of the $\mathrm{AW}(3)$ algebra (see 20, for details).

Now consider the case of the big -1 Jacobi polynomials and choose the following operators:

$$
X=L_{0}+\alpha+\beta+1, \quad Y=x, \quad Z=-\frac{2}{x}(c+(x-1)(x+c) R),
$$

where $L_{0}$ is the operator given by (2.10). 
It is then easy to verify that these operators satisfy the linear anticommutation relations

$$
X Y+Y X=Z+\omega_{3}, \quad Y Z+Z Y=\omega_{1}, \quad Z X+X Z=4 Y+\omega_{2},
$$

where

$$
\omega_{1}=-4 c, \omega_{2}=4(\alpha-\beta c), \omega_{3}=2(\beta-\alpha c) .
$$

The Casimir operator of the algebra defined by (6.4) is

$$
Q=Z^{2}+4 Y^{2} \text {. }
$$

In the realization (6.3) the Casimir operator takes the constant value $Q=4\left(c^{2}+1\right)$.

In this realization the operator $X$ (up to an additive constant) is the operator of which the polynomials $P_{n}^{(-1)}(x)$ are the eigenfunctions. The operator $Y$ here corresponds to multiplication by $x$.

The "dual" realization of the algebra (6.4) is obtained if one takes an infinite discrete basis $e_{n}, n=0,1,2, \ldots$ on which the operators $X, Y$ act as

$$
X e_{n}=\left(\lambda_{n}+\alpha+\beta+1\right) e_{n}, \quad Y e_{n}=u_{n+1}^{(-1)} e_{n+1}+b_{n}^{(-1)} e_{n}+e_{n-1},
$$

where $\lambda_{n}$ is the eigenvalue (2.17) and where the recurrence coefficients $u_{n}^{(-1)}, b_{n}^{(-1)}$ are given by (2.20), (2.21). Thus in this representation the operator $Y$ is a Jacobi (i.e. tri-diagonal) matrix and the eigenvalue equation

$$
Y \vec{P}=x \vec{P}
$$

is equivalent to the recurrence relation (2.22) for the big -1 Jacobi polynomials. Indeed, we can present the vector $\vec{P}$ in terms of its expansion coefficients over the basis $e_{n}$ :

$$
\vec{P}=\sum_{n=0}^{\infty} C_{n} e_{n} .
$$

Without loss of generality we can choose $C_{0}=1$. The coefficients $C_{n}$ in this expansion are then found to satisfy the recurrence relation (2.22) and it is seen moreover that these $C_{n}$ are monic polynomials in $x$ of degree $n$. Hence $C_{n}=$ $P_{n}^{(-1)}(x)$.

\section{TWO-DIAgONAL BASIS FOR THE OPERATOR $L_{0}$ AND A GENERALIZATION OF GAUSS' HYPERGEOMETRIC FUNCTIONS}

We already showed that the Dunkl-type operator $L_{0}$ is tri-diagonal in the ordinary monomial basis $x^{n}$ (see formula (2.15)). There exists, however, another polynomial basis in which the operator $L_{0}$ is two-diagonal. This basis can be constructed as follows:

$$
\begin{aligned}
\phi_{0}=1, \phi_{1}(x)=x-1, \phi_{2}(x)= & \left(x^{2}-1\right), \ldots, \\
\phi_{2 n}(x) & =\left(x^{2}-1\right)^{n}, \phi_{2 n+1}(x)=(x-1)\left(x^{2}-1\right)^{n} .
\end{aligned}
$$

It is easily verified that

$$
L_{0} \phi_{n}(x)=\lambda_{n} \phi_{n}(x)+\eta_{n} \phi_{n-1}(x),
$$

where $\lambda_{n}$ is the eigenvalue given by (2.17), and

$$
\eta_{n}=\left\{\begin{array}{c}
2 n(c-1), \quad \text { if } n \text { even, } \\
-2(c+1)(\alpha+n), \quad \text { if } n \text { odd }
\end{array}\right.
$$


Now consider the eigenvalue equation

$$
L_{0} P_{n}(x)=\lambda_{n} P_{n}(x)
$$

and expand the polynomials $P_{n}(x)$ over the basis $\phi_{n}(x)$ :

$$
P_{n}(x)=\sum_{s=0}^{n} A_{n s} \phi_{s}(x)
$$

For the expansion coefficients $A_{n s}$ we have from (7.3):

$$
A_{n, s+1}=\frac{A_{n s}\left(\lambda_{n}-\lambda_{s}\right)}{\eta_{s+1}} .
$$

From (7.4), the coefficients $A_{n s}$ can be found explicitly in terms of $A_{n 0}$ :

$$
A_{n s}=A_{n 0} \frac{\left(\lambda_{n}-\lambda_{0}\right)\left(\lambda_{n}-\lambda_{1}\right) \ldots\left(\lambda_{n}-\lambda_{s-1}\right)}{\eta_{1} \eta_{2} \ldots \eta_{s}}
$$

or in terms of the coefficient $A_{n n}$ :

$$
A_{n s}=A_{n n} \frac{\eta_{n} \eta_{n-1} \ldots \eta_{s+1}}{\left(\lambda_{n}-\lambda_{n-1}\right)\left(\lambda_{n}-\lambda_{n-2}\right) \ldots\left(\lambda_{n}-\lambda_{s}\right)} .
$$

We thus have the following explicit formula for the polynomials $P_{n}(x)$ :

$$
P_{n}(x)=A_{n 0} \sum_{s=0}^{n} \frac{\left(\lambda_{n}-\lambda_{0}\right)\left(\lambda_{n}-\lambda_{1}\right) \ldots\left(\lambda_{n}-\lambda_{s-1}\right)}{\eta_{1} \eta_{2} \ldots \eta_{s}} \phi_{s}(x) .
$$

Expression (7.7) resembles Gauss' hypergeometric function and can be considered as a nontrivial generalization of it. Indeed, products in the numerator and denominator of (7.7) can easily be presented in terms of ordinary Pochhammer symbols, and we thus recover the explicit formulas (2.24) and (2.25). Note, nevertheless, that the form (7.7) looks much simpler.

Moreover, also note that in the basis $\phi_{n}(x)$ the operators $X$ and $Y$ of the algebra defined by (6.3) and (6.4) become lower and upper triangular:

$$
X \phi_{n}(x)=\left(L_{0}+\alpha+\beta+1\right) \phi_{n}(x)=\left(\lambda_{n}+\alpha+\beta+1\right) \phi_{n}(x)+\eta_{n} \phi_{n-1}(x)
$$

and

$$
Y \phi_{n}(x)=x \phi_{n}(x)=\phi_{n+1}(x)+(-1)^{n} \phi_{n}(x) .
$$

Note that such bases in which the operators $X, Y$ are two-diagonal were central objects in Terwilliger's approach to Leonard pairs [17, [18]. Formulas similar to (7.7) also appear in the theory of Leonard pairs.

\section{ACKNOWLEDGMENTS}

The authors are indebted to R. Askey, C. Dunkl, T. Koornwinder, W. Miller, V. Spiridonov, P. Terwilliger and P. Winternitz for stimulating communications and to an anonymous referee for valuable remarks. The second author thanks CRM (U de Montréal) for its hospitality. 


\section{REFERENCES}

[1] G.E. Andrews and R. Askey, Classical orthogonal polynomials, Polynômes Orthogonaux et Applications, Lecture Notes in Mathematics, 1985, V. 1171, 36-62. MR838970 (88c:33015b)

[2] E. Bannai and T. Ito, Algebraic Combinatorics I: Association Schemes. Benjamin \& Cummings, Menlo Park, CA, 1984. MR882540 (87m:05001)

[3] Y. Ben Cheikh and M. Gaied, Characterization of the Dunkl-classical symmetric orthogonal polynomials, Appl. Math. and Comput. 187, (2007) 105-114. MR2323560 (2008c:33015)

[4] T. Chihara, An Introduction to Orthogonal Polynomials, Gordon and Breach, NY, 1978. MR0481884 (58:1979)

[5] L.M. Chihara and T.S. Chihara, A class of nonsymmetric orthogonal polynomials. J. Math. Anal. Appl. 126 (1987), 275-291. MR 900545 (88h:42023)

[6] C.F. Dunkl, Integral kernels with reflection group invariance. Canadian Journal of Mathematics, 43 (1991) 1213-1227. MR1145585 (93g:33012)

[7] Ya.L. Geronimus, On polynomials orthogonal with respect to the given numerical sequence and on Hahn's theorem, Izv.Akad.Nauk, 4 (1940), 215-228 (in Russian).

[8] J. Geronimo and W. Van Assche, Orthogonal polynomials on several intervals via a polynomial mapping, Trans. Amer. Math. Soc., 308(2) (1988), 559-581. MR.951620 (89f:42021)

[9] T. Ito and P. Terwilliger, Double Affine Hecke Algebras of Rank 1 and the $Z_{3}$-Symmetric Askey-Wilson Relations, SIGMA 6 (2010), Paper 065, 9 pages. MR2725018

[10] R. Koekoek and R. Swarttouw, The Askey-scheme of hypergeometric orthogonal polynomials and its q-analogue, Report no. 98-17, Delft University of Technology, 1998.

[11] R. Koekoek, P. Lesky, and R. Swarttouw, Hypergeometric Orthogonal Polynomials and Their q-analogues, Springer-Verlag, 2010. MR2656096 (2011e:33029)

[12] T. Koornwinder, On the limit from q-Racah polynomials to big q-Jacobi polynomials, ArXiv:1011.5585.

[13] A.B.J. Kuijlaars, A. Martinez-Finkelshtein, and R. Orive, Orthogonality of Jacobi polynomials with general parameters, Electronic Trans. Numer. Anal. 19 (2005), 1-17. MR2149265 (2006e:33010)

[14] D. Leonard, Orthogonal Polynomials, Duality and Association Schemes, SIAM J. Math. Anal. 13 (1982) 656-663. MR661597 (83m:42014)

[15] F. Marcellán and J. Petronilho, Eigenproblems for Tridiagonal 2-Toeplitz Matrices and Quadratic Polynomial Mappings, Lin. Alg. Appl. 260 (1997) 169-208. MR1448355 (98c:15032)

[16] G. Szegö, Orthogonal Polynomials, fourth edition, AMS, 1975. MR0372517 (51:8724)

[17] P. Terwilliger, Two linear transformations each tridiagonal with respect to an eigenbasis of the other. Linear Algebra Appl. 330 (2001) 149-203. MR1826654 (2002h:15021)

[18] P. Terwilliger, Two linear transformations each tridiagonal with respect to an eigenbasis of the other; an algebraic approach to the Askey scheme of orthogonal polynomials, arXiv:math/0408390v3. MR1826654 (2002h:15021)

[19] L.Vinet and A. Zhedanov, A "missing" family of classical orthogonal polynomials, J. Phys. A. 44 (2011), no. 8, 085201, 16pp. MR2770369 (2012c:42065)

[20] A. S. Zhedanov. Hidden symmetry of Askey-Wilson polynomials, Teoret. Mat. Fiz. 89 (1991) 190-204. (English transl.: Theoret. and Math. Phys. 89 (1991), 1146-1157). MR1151381 (93f:33019)

[21] A.S. Zhedanov, Rational spectral transformations and orthogonal polynomials, J. Comput. Appl. Math. 85, no. 1 (1997), 67-86. MR1482157(98h:42026)

Centre de recherches mathématiques, Université de Montréal, P.O. Box 6128, Centre-ville Station, Montréal, Québec, H3C 3J7 Canada

Institute for Physics And Engineering, R. Luxemburg str. 72, 83114 Donetsk, Ukraine 